\title{
The Effect of the Earth Matter on Three Neutrino Oscillations and Sensitivity to CP Phase Parameter
}

\author{
Bushra Shafad* and Faisal Akram + \\ Centre For High Energy Physics, Punjab University, Lahore(54590), Pakistan.
}

(Dated: October 31, 2018)

\begin{abstract}
We find an analytical expression of neutrino evolution operator in the Earth matter using perturbative approach in the context of three neutrino oscillations. We find that our analytical expression is highly accurate by comparing its results with the numerical solutions of neutrino evolution equation at energy scales relevant for solar, reactor, atmospheric, and accelerator neutrinos. Using our analytical approach we study the accuracy of hypothesis of treating the Earth density piecewise constant. We also study how the Earth matter effect can change the sensitivity to CP phase parameter $\delta_{C P}$. Through nadir angle averaged conversion probabilities of neutrino and anti-neutrino, we find that the sensitivity to $\delta_{C P}$ is maximum in the energy range 0.2 to $1 \mathrm{GeV}$ and through energy averaged conversion probabilities, we find that the sensitivity is maximum about nadir angle $72.5^{\circ}$ for neutrinos oscillating in the Earth matter.

PACS numbers: 14.60.Pq, 26.65.+t, 13.15.+g, 91.35.-x
\end{abstract}

*Electronic address: bushra.chep@pu.edu.pk:

${ }^{\dagger}$ Electronic address: faisal.chep@pu.edu.pk; 


\section{INTRODUCTION}

Solar neutrino problem (SNP) came into sight in 1968 when Homestake chlorine experiment [1] found that the measured flux of solar neutrinos $v_{e}$ was significantly smaller than predicted by the standard solar model (SSM). An elegant way to explain the observed depletion, already proposed by Pontecorvo [2], was the neutrino oscillations in which a neutrino of one flavor transforms into another while propagating in free space. This lead to an extensive experimental program in neutrino physics to confirm the depletion and hypothesis of neutrino oscillations. The subsequent Ga experiments (SAGE [3]], GALLEX [4], and GNO [5]), which like Homestake also measured the flux of solar neutrinos $v_{e}$ through charge current (CC) weak interaction, confirmed the depletion. Kamiokande [6] and later SuperKamiokande (SK) [7-11] experiments pioneered in real-time solar neutrinos observation and provided the direct evidence that solar neutrinos are coming from the direction of sun, a feature which greatly helped in separating the signal from background. Both Kamiokande and SK measured solar neutrinos through elastic scattering process $v_{x}+e \rightarrow v_{x}+e$, which is sensitive to all flavors of active neutrinos $(x=e, \mu$, and $\tau)$, however sensitivity to $\nu_{\mu}$ and $\nu_{\tau}$ is reduced because $\sigma\left(v_{\mu, \tau} e\right) \approx 0.16 \sigma\left(v_{e} e\right)$. Consequently the detectors were unable to measure total flux of all flavors of neutrinos coming from the direction of sun, a quantity which was crucial to confirm the hypothesis of neutrino oscillations. The direct confirmation of solar neutrino hypothesis was provided by a new real-time solar neutrino experiment SNO (Sudbury Neutrino Observatory) [12], when it published the result [13] of ${ }^{8} \mathrm{~B}$ solar neutrinos flux measured by neutral current (NC) weak interaction process $\left(v_{x}+d \rightarrow v_{x}+p+n\right)$. This NC process is equally sensitive to all neutrino flavors, hence the measured flux is sum of the flux of all neutrino flavors. The agreement with SSM prediction provided a conclusive prove of neutrino oscillations in solar neutrinos. The next step was to determine neutrino oscillations parameters. Neutrino oscillations can occur if it is assumed that flavor states (the states which take part in $\mathrm{CC}$ and $\mathrm{NC}$ weak interactions) are different from mass eigen states and at least one neutrino is massive. The unitary matrix which relates flavor and mass eigen states is defined through 4 parameters, in which 3 are the angles $\left(\theta_{12}, \theta_{23}, \theta_{13}\right)$

and one is the CP phase parameter $\delta_{C P}$ [14], assuming neutrino are Dirac particles. The survival or conversion probabilities of neutrinos additionally depend on two independent squared mass differences $\left(\Delta m_{21}^{2}, \Delta m_{31}^{2}\right)$. So there are total six constants which describe 
neutrino oscillations. In case where only two flavor of neutrinos are assumed, the number the independent parameters are reduced to two; one mixing angle and one squared mass difference. To calculate solar neutrino $\nu_{e}$ data, we merely require the knowledge of averaged electron neutrino survival probability, which in three neutrino oscillations depends on the mixing angles $\theta_{12}$ and $\theta_{13}$, and squared mass difference $\Delta m_{21}^{2}$. The global analysis of solar neutrino data of SK+SNO experiments [15] showed, LMA (large mixing angle) solution with $\Delta m_{21}^{2}=4.8 \times 10^{-5} \mathrm{eV}^{2}, \sin ^{2} \theta_{12}=0.31$ is the best solution. However, other solutions were not ruled out with sufficient statistical significance. In the Ref. [15] the constraint $\sin ^{2} \theta_{13}=0.0219$ coming from reactor neutrino experiments is assumed, which shows that dependence of survival probability on $\theta_{13}$ is extremely weak so that effectively 2 neutrino oscillations are sufficient to describe solar neutrino data. The issue of finding unique solution of mixing parameters was resolved by KamLAND experiment of reactor anti-neutrinos $\bar{\nu}_{e}$, which is sensitive to $\Delta m^{2} \sim 10^{-5}$. The global analysis of solar neutrino data of SK+SNO and KamLAND experiment [16] finally identified the LMA solution with $\Delta m_{21}^{2}=7.49 \times 10^{-5}$ $\mathrm{eV}^{2}, \sin ^{2} \theta_{12}=0.307$ as a true solution of solar neutrino problem. Neutrino oscillations are also studied in atmospheric neutrino experiments. Atmospheric neutrinos predominantly $v_{\mu}$ and $\bar{v}_{\mu}$ are produced by the decay of $\pi$ and $K$ mesons, which are provided by primary cosmic rays in upper atmosphere. Neutrino oscillations in the atmospheric neutrinos were first discovered by observing muon neutrinos disappearance by SK [17]. Later experiments, MACRO [18], Soudan 2 [19], and MINOS [20] confirmed the observation. Unfortunately their exist large uncertainties in the predictions of atmospheric neutrinos flux, which makes it difficult to precisely determine the neutrino oscillation parameters. More recently the muon disappearance is also observed in long-baseline accelerator neutrino $\nu_{\mu}$ experiments, $\mathrm{K} 2 \mathrm{~K}$ [21], MINOS+ [22], T2K [23], and NO $\nu \mathrm{A}$ [24]. Electron neutrino appearance $\nu_{\mu} \rightarrow \nu_{e}$ is measured by MINOS [25], whereas anti-electron appearance $\bar{\nu}_{\mu} \rightarrow \bar{\nu}_{e}$ is meaured by T2K [26]. These measurements allow precise determination of the parameters $\theta_{23}$ and $\Delta m_{32}^{2}$. There exist several different analysis, here we report the fitted values given in PDG [27]; $\sin ^{2} \theta_{23}=0.417_{-0.028}^{+0.025}, \Delta m_{32}^{2}=2.51 \pm 0.05\left(10^{-3} \mathrm{eV}^{2}\right)$, assuming normal mass hierarchy (i.e., $\left.m_{1}<m_{2}<m_{3}\right)$. The parameter $\sin ^{2} \theta_{13}$ is extracted from the measured disappearance of anti-neutrinos $\bar{\nu}_{e}$ in reactor neutrinos experiments (Double Chooz [28], RENO [29], and Daya Bay [30]), at relatively small distance $L \sim 1 \mathrm{~km}$ corresponding to large value of $\Delta m_{32}^{2}$. Fitted average value reported in PDG is $\sin ^{2} \theta_{13}=2.12 \pm 0.08\left(10^{-2}\right)$. Having relatively pre- 
cise knowledge of neutrino oscillations parameters, the interest is now shifted to CP-phase $\delta_{C P}$. Recently $\delta_{C P}=1.45_{-0.26}^{+0.27} \pi$ is measured by $\mathrm{T} 2 \mathrm{~K}$ experiment [31] through the study of difference between conversion probabilities of $v_{\mu} \rightarrow v_{e}$ and $\bar{v}_{\mu} \rightarrow \bar{v}_{e}$ using accelerator neutrinos.

Although neutrinos are weakly interacting particles, their propagation through matter significantly modifies the neutrino oscillations through coherent interaction with matter electrons via charge changing (CC) weak interaction [33]. In case of matter of constant density, the parameters of oscillations are effectively changed, depending upon the value of energy and density. Dependence of effective oscillating parameters on energy and density has a resonance character [33], which can lead to a strong enhancement of the oscillations, independent of the values of vacuum mixing angles. However, when conversion probability is averaged over energy, the maximum depletion which it can yield is $1 / 2$. A large observed depletion (less than 1/2) in solar neutrinos $\nu_{e}$ flux is the consequence of MSW (MikheyevSmirnov-Wolfenstein) effect [34] which occurs in a slowly varying density, while crossing the point where resonance condition is satisfied in the interior of Sun. MSW effect, produced by the Earth's variable density, is not relevant for solar, atmospheric, accelerator, and reactor neutrinos, as for reactor and solar neutrinos resonance density become very large and for atmospheric and accelerator neutrinos it becomes very small as compared to electron density in the Earth. Nevertheless it is found that the Earth's density profile can significantly affect the neutrino oscillations. For atmospheric or accelerator neutrinos, having energies $E \gtrsim 2$ $\mathrm{GeV}$, the effect of relatively small value of $\Delta m_{21}^{2}=7.49 \times 10^{-5} \mathrm{eV}^{2}$ can be neglected to leading order, consequently the Earth matter strongly suppresses the oscillations due to $\Delta m_{21}^{2}$. In this case three neutrino oscillations probabilities are effectively described by two neutrino oscillations. The relevant oscillation parameters are $\theta_{13}, \Delta m_{31}$, and Earth electron density $N_{e}$. It is shown in Refs. [35-37], the probability $P\left(v_{e} \rightarrow v_{\mu(\tau)}\right)$ is maximally enhanced for neutrino of energy $E \simeq 7.1 \mathrm{GeV}$ and traveling the path length $L \simeq 11740 \mathrm{~km}$ for $\Delta m_{31}^{2} \simeq 2.5 \times 10^{-3} \mathrm{eV}^{2}, \sin ^{2} 2 \theta_{13} \simeq 0.09$, and $N_{e}=2.2 N_{A} \mathrm{~cm}^{-3}$. The result is established by using the analytical solution of neutrino evolution equation through the Earth. This parametric enhancement due to the effect of the Earth matter can significantly amplify oscillation probabilities for both atmospheric and accelerator neutrinos as the parametric values are close to LMA solution. It is also noted that this resonance like effect, though amplifying the conversion $v_{e} \rightarrow v_{\mu(\tau)}$, suppresses $\bar{v}_{e} \rightarrow \bar{v}_{\mu(\tau)}$ if $\Delta m_{31}^{2}>0$. For $\Delta m_{31}^{2}<0$, the 
effect is reversed and amplification is produced for $\bar{v}_{e} \rightarrow \bar{v}_{\mu(\tau)}$. Solar neutrinos can also be affected by the coherent interaction with the Earth matter. Solar neutrinos detected at night time reach the detector after passing through the Earth interior, which produces a small enhancement in $\nu_{e}$ flux through $v_{\mu(\tau)} \rightarrow v_{e}$. This regeneration effect for solar neutrinos has been observed in SK, SNO, and BOREXINO [38, 39] through non-zero value of day-night asymmetry $A_{D-N}$ of measured event rate.

The effect of parametric enhancement is studied using analytical expression of neutrino oscillations obtained by treating the Earth density piecewise constant. Usually divided into two regions; core $(0 \leq r<3485 \mathrm{~km})$ and mantle $(3486 \leq r<6371 \mathrm{~km})$. In this work we

obtain an analytical expression of neutrino evolution operator treating the Earth density piecewise variable in five shells. For each shell the variation is treated perturbatively about its average value. This scheme is also adopted in Ref. [40], where the problem is solved for two neutrino oscillations. Our analytical expressions agree with numerical solutions of three neutrino evolution equation in the Earth at energies relevant for solar, reactor, atmospheric, and accelerator neutrinos. We also study, how the Earth matter effect can change the sensitivity to $\mathrm{CP}$ phase parameter $\delta_{C P}$

In Sec. II, we describe the general formalism of 3 neutrino oscillations. In Sec. III, we discuss the solution of evolution equation in matter of constant density. In Sec. IV, we discuss the parametrization of radial profile of electron density in the Earth. In Sec. V, we apply perturbation theory to obtain solution of evolution operator. In Sec. VI, we discuss accuracy of our analytical expressions and study the effect of the Earth matter on neutrino oscillations and sensitivity to CP phase.

\section{THE GENERAL FORMULISM}

Neutrino flavor states $\left|\nu_{\alpha}\right\rangle$ are written as linear combinations of mass eigen states $\left|\nu_{k}\right\rangle$ [2] via a unitary matrix, called Pontecorvo-Maki-Nakagawa-Sakata (PMNS) matrix, as following

$$
\left|\nu_{\alpha}\right\rangle=\sum_{k=1}^{3} U_{\alpha k}^{*}\left|\nu_{k}\right\rangle,
$$


where $\alpha=e, \mu, \tau$ and $k=1,2,3$. We use the following parametrization of PMNS matrix [32]

$$
U=\left(\begin{array}{ccc}
c_{12} c_{13} & s_{12} c_{13} & s_{13} e^{i \delta} \\
-s_{12} c_{23}-c_{12} s_{23} s_{13} e^{-i \delta} & c_{12} c_{23}-s_{13} s_{13} s_{23} e^{-i \delta} & c_{13} s_{23} \\
s_{12} s_{23}-c_{12} s_{13} c_{23} e^{-i \delta} & -c_{12} s_{23}-s_{12} s_{13} c_{23} e^{-i \delta} & c_{13} c_{23}
\end{array}\right)
$$

where $c_{i j}=\cos \theta_{i j}, s_{i j}=\sin \theta_{i j}$ for $i=1,2,3$ and $\delta_{C P}$ is the Dirac CP phase. For antineutrinos, CP phase is replaced by $-\delta_{C P}$. An arbitrary neutrino state $|\psi(t)\rangle$ can be expressed in terms of both flavor or mass eigen states.

$$
|\psi(t)\rangle=\sum_{\alpha} \psi_{\alpha}(t)\left|\nu_{\alpha}\right\rangle=\sum_{k} \psi_{k}(t)\left|\nu_{k}\right\rangle
$$

where $\psi_{\alpha}(t)$ and $\psi_{k}(t)$ are the components of the state $|\psi(t)\rangle$ in flavor and mass basis respectively and they are related as

$$
\psi_{k}(t)=\sum_{\alpha} U_{\alpha k}^{*} \psi_{\alpha}(t)
$$

In matrix form Eq. 4 is written as following

$$
\psi^{f}(t)=U \psi^{m}(t)
$$

where

$$
\psi^{f}(t)=\left(\begin{array}{c}
\psi_{e}(t) \\
\psi_{\mu}(t) \\
\psi_{\tau}(t)
\end{array}\right), \quad \psi^{m}(t)=\left(\begin{array}{c}
\psi_{1}(t) \\
\psi_{2}(t) \\
\psi_{3}(t)
\end{array}\right)
$$

In mass basis, the vacuum Hamiltonian is

$$
H_{0}^{(m)}=\left(\begin{array}{ccc}
E_{1} & 0 & 0 \\
0 & E_{2} & 0 \\
0 & 0 & E_{3}
\end{array}\right)
$$

Whereas in flavor basis it is given by $H_{0}^{(f)}=U H_{0}^{(m)} U^{\dagger}$. As neutrinos are relativistic, having very small masses as compared to their energies, so the energy $E_{i} \simeq p+\frac{m_{i}^{2}}{2 p}$. Subtracting the constant $p+\frac{m_{1}^{2}}{2 p}$ from the diagonal of $H_{0}^{(m)}$

$$
H_{0}^{(m)}=\frac{1}{2 p}\left(\begin{array}{ccc}
0 & 0 & 0 \\
0 & \Delta m_{21}^{2} & 0 \\
0 & 0 & \Delta m_{31}^{2}
\end{array}\right)
$$


where $\Delta m_{i j}^{2}=m_{i}^{2}-m_{j}^{2}$ and $E \simeq p$. When neutrinos are propagating through matter, the total Hamiltonian in flavor basis is sum of vacuum Hamiltonian $H_{0}^{(f)}$ and an interacting part $H_{I}^{(f)}(x)$.

$$
H^{(f)}(x)=H_{0}^{(f)}+H_{I}^{(f)}(x)
$$

Flavor states of neutrinos $\left(\nu_{e}, \nu_{\mu}\right.$, and $\left.\nu_{\tau}\right)$ interact with the electrons in the matter through charge current (CC) and neutral current (NC) weak interactions. Since only $\nu_{e}$ neutrinos take part in CC interaction and all flavors are equally sensitive to NC interaction, therefore NC interaction amplitudes do not contribute to $H_{I}^{(f)}(x)$. It is noted that decoherent interaction, in which the state of incoming neutrino is changed, has negligible effect on the propagation of solar, atmospheric, accelerator, and reactor neutrinos. The interaction Hamiltonian is, therefore, given as

$$
H_{I}^{(f)}(x)=A(x)\left(\begin{array}{lll}
1 & 0 & 0 \\
0 & 0 & 0 \\
0 & 0 & 0
\end{array}\right)
$$

where $A(x)=2 \sqrt{2} G_{F} N_{e}(x)$, in which $G_{F}$ is Fermi coupling constant and $N_{e}(x)$ is electron density in matter. For anti-neutrinos the sign of electron density is inverted in the interaction Hamiltonian.

For calculational convenience we also convert $H^{(f)}(x)$ into a traceless matrix $\tilde{H}^{(f)}(x)$ defined as following

$$
\tilde{H}^{(f)}(x)=H^{(f)}(x)-\frac{1}{3} \operatorname{Tr}\left(H^{(f)}(x)\right) I
$$

where

$$
\operatorname{Tr}\left(H^{(f)}(x)\right)=\frac{1}{2 p}\left(\Delta m_{21}^{2}+\Delta m_{31}^{2}\right)+A(x) .
$$

\section{SOLUTION OF TIME EVOLUTION OPERATOR IN A CONSTANT DEN- SITY MATTER}

The time evolution equation of neutrino in matter is given as

$$
\frac{i \partial \psi^{f}(t)}{\partial t}=\tilde{H}^{(f)}(x) \psi^{f}(t)
$$

In matter of constant density, $\tilde{H}^{(f)}$ does not depends upon $x$ so its solution is given as

$$
\psi^{f}(t)=e^{-i \tilde{H}^{(f)} t} \psi^{f}(0)
$$


Neutrinos travel with speed very close to the speed of light so $t=x$. Thus the time evolution operator is given by

$$
\mathcal{U}(x)=e^{-i \tilde{H}^{(f)} x}
$$

In order to obtain a computationally useful expression of $\mathcal{U}(x)$, we follow the Ref. [41], in which $\tilde{H}^{(f)}$ is decomposed into linear combination of Gell-Mann matrices $\left(\lambda_{j}\right)$ as following

$$
\tilde{H}^{(f)}=h_{j} \lambda_{j},
$$

where $h_{j}=\frac{1}{2} \operatorname{Tr}\left(\tilde{H}^{(f)} \lambda_{j}\right)$ are real coefficients obtained by applying orthonormalization condition $\operatorname{Tr}\left[\lambda_{i} \lambda_{j}\right]=2 \delta_{i j}$. Similarly time evolution operator can also be expresses as a linear combination of Gell-Mann matrices and identity matrix, which is required because $\mathcal{U}(x)$ is not traceless.

$$
\mathcal{U}(x)=u_{0} I+i u_{j} \lambda_{j},
$$

where $u_{0}=\frac{1}{3} \operatorname{Tr}[\mathcal{U}(x)]$ and $u_{j}=\frac{1}{2 i} \operatorname{Tr}\left[\mathcal{U}(x) \lambda_{j}\right]$ are again obtained by using the orthonormalization condition of Gell-Man matrices. These coefficients can be expressed in terms of eigen values of $\tilde{H}^{(f)}$, which we represent by $E_{\alpha}^{(m)}$, as following.

$$
\begin{aligned}
& u_{0}=\frac{1}{3} \sum_{\alpha=1}^{3} e^{-i E_{\alpha}^{(m)} x} \\
& u_{j}=-\frac{i}{2} \sum_{a=1}^{3} \frac{\partial E_{\alpha}^{(m)}}{\partial h_{j}} e^{-i E_{\alpha}^{(m)} x},
\end{aligned}
$$

where the second equation is obtained by using $\frac{\partial \mathcal{U}}{\partial h_{j}}=-i x \mathcal{U} \lambda_{j}$. The eigen values of the matrix $\tilde{H}^{(f)}$ are given by following characteristic polynomial equation.

$$
E_{\alpha}^{(m) 3}+a_{2} E_{\alpha}^{(m) 2}+a_{1} E_{\alpha}^{(m)}+a_{0}=0
$$

where $a_{2}=-\operatorname{Tr}\left[\tilde{H}^{(f)}\right]=0, a_{1}=-\frac{1}{2}\left(\operatorname{Tr}\left[\tilde{H}^{(f)}\right]^{2}-\operatorname{Tr}\left[\left(\tilde{H}^{(f)}\right)^{2}\right]\right)=h_{i} h_{i} \equiv|h|^{2}$, and $a_{0}=-\operatorname{det}\left[\tilde{H}^{(f)}\right]=-\frac{2}{3} d_{j k l} h_{j} h_{k} h_{l}$, in which totally symmetric tensor $d_{j k l}=\frac{1}{4} \operatorname{Tr}\left[\left\{\lambda_{j}, \lambda_{k}\right\} \lambda_{l}\right]$. Differentiating the characteristic equation with respect to $h_{j}$ to yield $\frac{\partial E_{\alpha}^{(m)}}{\partial h_{j}}$, given as

$$
\frac{\partial E_{\alpha}^{(m)}}{\partial h_{j}}=\frac{2\left(E_{\alpha}^{(m)} h_{j}+[h * h]_{j}\right)}{3\left(E_{\alpha}^{(m)}\right)^{2}-|h|^{2}},
$$

where $[h * h]_{j}=d_{j k l} h_{k} h_{l}$. Using it in Eq. (18b) to obtain $u_{j}$ and then $\mathcal{U}(x)$ from Eq. (17)

$$
\mathcal{U}(x)=\frac{1}{3} \sum_{\alpha=1}^{3} e^{-i E_{\alpha}^{(m)} t} A_{\alpha},
$$


where $A_{\sigma}=\left(I+\rho_{\alpha} \chi_{\alpha j} \lambda_{j}\right), \rho_{\alpha}=\frac{2}{3 E_{\alpha}^{(m)}-|h|^{2}}$ and $\chi_{\alpha j}=E_{\alpha}^{(m)} h_{j}+[h * h]_{j}$. For completeness we also give the solution of characteristic equation

$$
\begin{aligned}
& E_{1}^{(m)}=-\sqrt{\frac{a_{1}}{3}} \cos \left[\frac{1}{3} \tan ^{-1}\left(\frac{1}{a_{0}} \sqrt{-a_{0}^{2}+\frac{4 a_{1}^{3}}{27}}\right)\right]+\sqrt{a_{1}} \sin \left[\frac{1}{3} \tan ^{-1}\left(-\frac{1}{a_{0}} \sqrt{a_{0}^{2}+\frac{4 a_{1}^{3}}{27}}\right)\right] \\
& E_{2}^{(m)}=-\sqrt{\frac{a_{1}}{3}} \cos \left[\frac{1}{3} \tan ^{-1}\left(\frac{1}{a_{0}} \sqrt{-a_{0}^{2}+\frac{4 a_{1}^{3}}{27}}\right)\right]-\sqrt{a_{1}} \sin \left[\frac{1}{3} \tan ^{-1}\left(-\frac{1}{a_{0}} \sqrt{a_{0}^{2}+\frac{4 a_{1}^{3}}{27}}\right)\right] \\
& E_{3}^{(m)}=2 \sqrt{\frac{a_{1}}{3}} \cos \left[\frac{1}{3} \tan ^{-1}\left(\frac{1}{a_{0}} \sqrt{-a_{0}^{2}+\frac{4 a_{1}^{3}}{27}}\right)\right]
\end{aligned}
$$

Notice that $\sum_{\alpha=1}^{3} E_{\alpha}^{(m)}=0$, which follows from the fact that $\tilde{H}^{(f)}$ is traceless. The eigen values given by Eqs. (22) can be used in Eq. (21) along with $h_{j}=\frac{1}{2} \operatorname{Tr}\left(\tilde{H}^{(f)} \lambda_{j}\right)$ to determine the evolution operator $\mathcal{U}(x)$ for the case of constant density.

\section{PARAMETERIZING THE EARTH ELECTRON DENSITY}

Electron density in the Earth is provided by Preliminary Earth Reference Model (PREM) [42], which divides the Earth interior into 8 shells of continuous density. We follow the scheme of Ref. [40] in which 4 outer shells are grouped into single shell and the density in each shell is fitted using following polynomial

$$
N(r)=\alpha_{k}+\beta_{k} r^{2}+\gamma_{k} r^{4}
$$

where $k=1$ to 5 , are the labels of the shells. The fitted values of the coefficients are given in Ref. [40] and we summarized them in Table 1. The functional form of Eq. (23) is invariant for non radial neutrino trajectory (i.e., nadir angle $\eta \neq 0$ )

$$
N_{k}(x)=\alpha_{k}^{\prime}+\beta_{k}^{\prime} x^{2}+\gamma_{k}^{\prime} x^{4}
$$

where

$$
\begin{aligned}
& \alpha_{k}^{\prime}=\alpha_{k}+\beta_{k} \sin ^{2} \eta+\gamma_{k} \sin ^{4} \eta, \\
& \beta_{k}^{\prime}=\beta_{k}+2 \gamma_{k} \sin ^{2} \eta, \\
& \gamma_{k}^{\prime}=\gamma_{k} .
\end{aligned}
$$

The trajectory coordinate $x$ of neutrino and nadir angle $\eta$ are defined in the Fig. 1 . In each shell the density is split as following

$$
N_{k}(x)=\bar{N}_{k}+\delta N_{k}(x)
$$


where $\bar{N}_{k}$ is the ( $\eta$ dependent) average density along the shell chord and $\delta N_{k}(x)$ is the residual density, which can be obtained by using equation (26) itself for given $\bar{N}_{k}$ obtained as following

$$
\bar{N}_{k}=\int_{x_{k}-1}^{x_{k}} d x N_{k}(x) /\left(x_{k}-x_{k}-1\right)
$$

\begin{tabular}{|c|c|c|c|c|c|}
\hline$k$ & Shell & {$\left[r_{k-1}, r_{r}\right]$} & $\alpha_{k}$ & $\beta_{k}$ & $\gamma_{k}$ \\
\hline 1 & Inner core & {$[0,0.192]$} & 6.099 & -4.119 & 0.00 \\
2 & Outer core & {$[0.192,0.546]$} & 5.803 & -3.653 & -1.086 \\
3 & Lower mantle & {$[0.546,0.895]$} & 3.156 & -1.459 & 0.280 \\
4 & Transition & {$[0.895,0.937]$} & -5.376 & 19.210 & -12.520 \\
5 & Upper mantle & {$[0.937,1]$} & 11.540 & -20.280 & 10.410 \\
\hline
\end{tabular}

TABLE I: Coefficients of the electron density parametrization $N_{k}(r)=\alpha_{k}+\beta_{k} r^{2}+\gamma_{k} r^{4}$ in $\mathrm{mol} / \mathrm{cm}^{3}$, for the different shells. The radial distance $r$ is normalized to the Earth radius. The fitted values are taken from Ref. [40].

\section{APPLICATION OF PERTURBATION THEORY}

In this section we present the analytic expression of neutrino evolution operator $\mathcal{U}(x)$ for propagation of neutrino through the Earth interior. Using equation (26), we split the Hamiltonian $\tilde{H}^{(f)}(x)$ given in equation (11) for the $k$ th shell as following

$$
\tilde{H}^{(f)}(x)=\bar{H}+\delta \tilde{H}^{(f)}(x)
$$

where $\bar{H}=\left.\tilde{H}^{(f)}\right|_{N_{e}(x) \rightarrow \bar{N}_{k}}$ and $\delta \tilde{H}^{(f)}(x)$ is

$$
\delta \tilde{H}^{(f)}(x)=\frac{2}{3} \sqrt{2} G_{F} \operatorname{Diag}\left[2 \delta N_{k}(x),-\delta N_{k}(x),-\delta N_{k}(x)\right] .
$$

This splitting insure that the constant part of the Hamiltonian $\bar{H}$ is traceless so that we could use the expressions of constant density given in Sec. III. Since the variation of density in each shell is relatively small so we can treat $\delta \tilde{H}^{(f)}(x)$ part of Hamiltonian as a perturbation and use the following perturbative solution of evolution operator of Eq. (13) for $k$ th shell

$$
\mathcal{U}\left(x_{k}-x_{k-1}\right)=e^{-i \bar{H}\left(x_{k}-x_{k-1}\right)}-i \int_{x_{k-1}}^{x_{k}} d x e^{-i \bar{H}\left(x_{k}-x\right)} \delta \tilde{H}^{(f)}(x) e^{-i \bar{H}\left(x-x_{k-1}\right)} .
$$




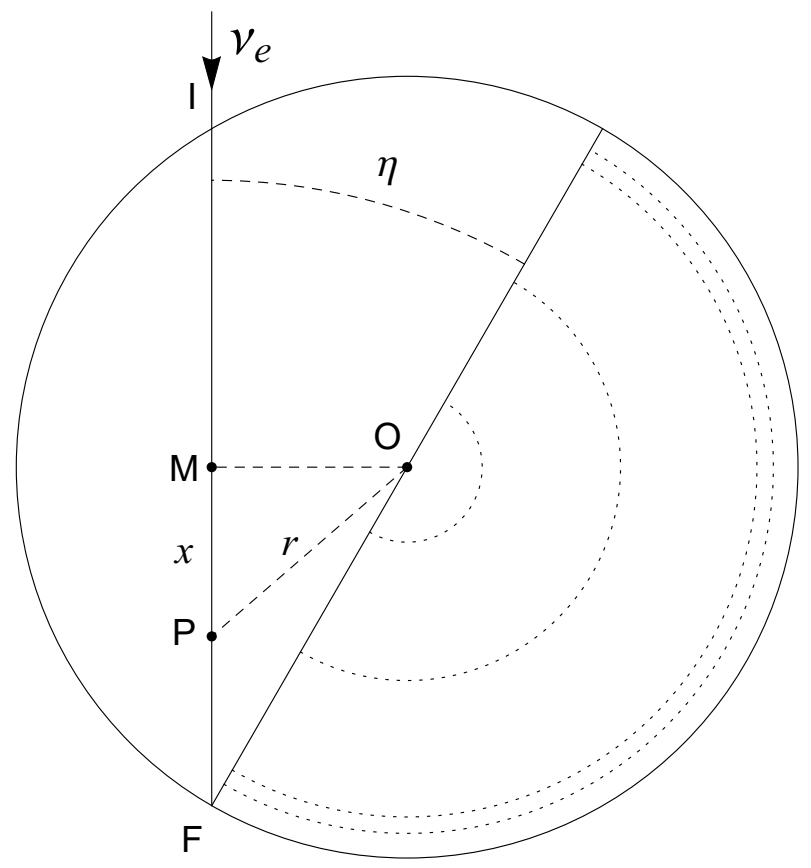

FIG. 1: Cross section of the Earth showing five shells. I and F are entering and exit points of neutrino trajectory respectively, $\mathrm{M}$ is the trajectory mid point, $r$ is the radial distance, and $x$ is distance from mid point $\mathrm{M}$.

Using Eq. (21), which gives evolution operator for constant density, we can calculate the exponential factors containing constant density Hamiltonian $\bar{H}_{k}$ in equation (30). The resultant expression is given by

$\mathcal{U}\left(x_{k}-x_{k-1}\right)=\frac{1}{3} \sum_{\alpha=1}^{3} e^{-i E_{\alpha}^{(m)}\left(x_{k}-x_{k-1}\right)} A_{\alpha}-\frac{i}{9} \sum_{\alpha, \beta} \int_{x_{k}-1}^{x_{k}} d x e^{-i E_{\alpha}^{(m)}\left(x_{k}-x\right)} A_{\alpha} \delta \tilde{H}^{(f)}(x) A_{\beta} e^{-i E_{\beta}^{(m)}\left(x-x_{k-1}\right)}$.

Using this equation we can calculate evolution operator of neutrino evolution in each shell. Total evolution operator is obtained from the product of evolution operators for all shells coming in neutrino trajectory for any given $\eta$, as following

$$
\mathcal{U}\left(x_{F}, x_{I}\right)=\prod_{k} \mathcal{U}\left(x_{k}-x_{k-1}\right)
$$

The expression (31) is our main result of evolution operator for three neutrino oscillations through the Earth interior. 


\section{RESULTS AND DISCUSSION}

Using the analytical expression of evolution operator through Earth interior, given in Eq. (32), we can calculate the probability $P\left(v_{\alpha} \rightarrow v_{\beta}\right)=\left|\mathcal{U}_{\beta \alpha}\left(x_{F}, x_{I}\right)\right|^{2}$ for given nadir angle and oscillations parameters that include three mixing angles $\left(\theta_{12}, \theta_{23}, \theta_{13}\right)$, one Dirac CP phase $\delta_{C P}$, and two independent squared mass differences $\left(\Delta m_{21}^{2}, \Delta m_{32}^{2}\right)$. In this section we compare the results of analytical expression with the solutions obtained by numerically solving the evolution Eq. (13) through the Earth interior. For comparison, we use three neutrino oscillation parameters of LMA solution (i.e., $\sin ^{2} \theta_{12}=0.307, \sin ^{2} \theta_{23}=0.417, \sin ^{2} \theta_{13}=$ $\left.2.12 \times 10^{-2}, \Delta m_{21}^{2}=7.49 \times 10^{-5} \mathrm{eV}^{2}, \Delta m_{32}^{2}=2.51 \times 10^{-3} \mathrm{eV}^{2}, \delta_{C P} \approx 1.45 \pi\right)$. For solar neutrinos $v_{e}$ the effect of the Earth matter interaction can be described by day-night asymmetry of average electron survival probability. For 3 neutrinos the day-night difference of averaged electron neutrino survival probability is given by

$$
P_{N}-P_{D}=-\cos ^{2} \theta_{13} \overline{\cos 2 \widehat{\theta}_{12}}\left(\left\langle P_{2 e}^{\oplus}\right\rangle-P_{2 e}^{(0)}\right)
$$

where $\cos 2 \widehat{\theta}_{12}$ is the effective value of $\cos 2 \theta_{12}$ at the production point of solar neutrino and bar stands for production point averaged value, $P_{2 e}^{(0)}=\left|U_{e 2}\right|^{2}$, and $\left\langle P_{2 e}^{\oplus}\right\rangle$ is the conversion probability of $v_{2} \rightarrow v_{e}$ through Earth interior averaged over nadir angle, as following

$$
\left\langle P_{2 e}^{\oplus}\right\rangle=\int_{0}^{\pi / 2} d \eta W(\eta) P_{2 e}^{\oplus},
$$

where $W(\eta)$ is nadir angle distribution function, whose functional form depends on latitude of the detector. We use the analytical expression of $W(\eta)$ given in Ref. [40]. The Eq. (33) shows that the effect of the Earth matter interaction is described by non-zero value of

$P_{2 e}^{\oplus}-P_{2 e}^{(0)}$. In Fig. 2, the plot of $P_{2 e}^{\oplus}-P_{2 e}^{(0)}$ versus $\cos \eta$ is given at $E=10 \mathrm{MeV}$. The solid curve in the figure represents the solution obtained by numerically solving neutrino evolution equation and marker symbols represent the results obtained from analytical expression. Disk (red) and rectangular (green) marks represent values with and without first order correction respectively. It is noted that the first order correction include the effect of variation of the Earth density in each shell, whereas in the results without the correction Earth density in each shell in treated constant. The comparison of the results given in Fig. 2 shows that constant density approximation fails for $\cos \eta>0.5$, i.e., when neutrinos total path length in the Earth is greater than its radius. The plot also shows that our analytical expression 


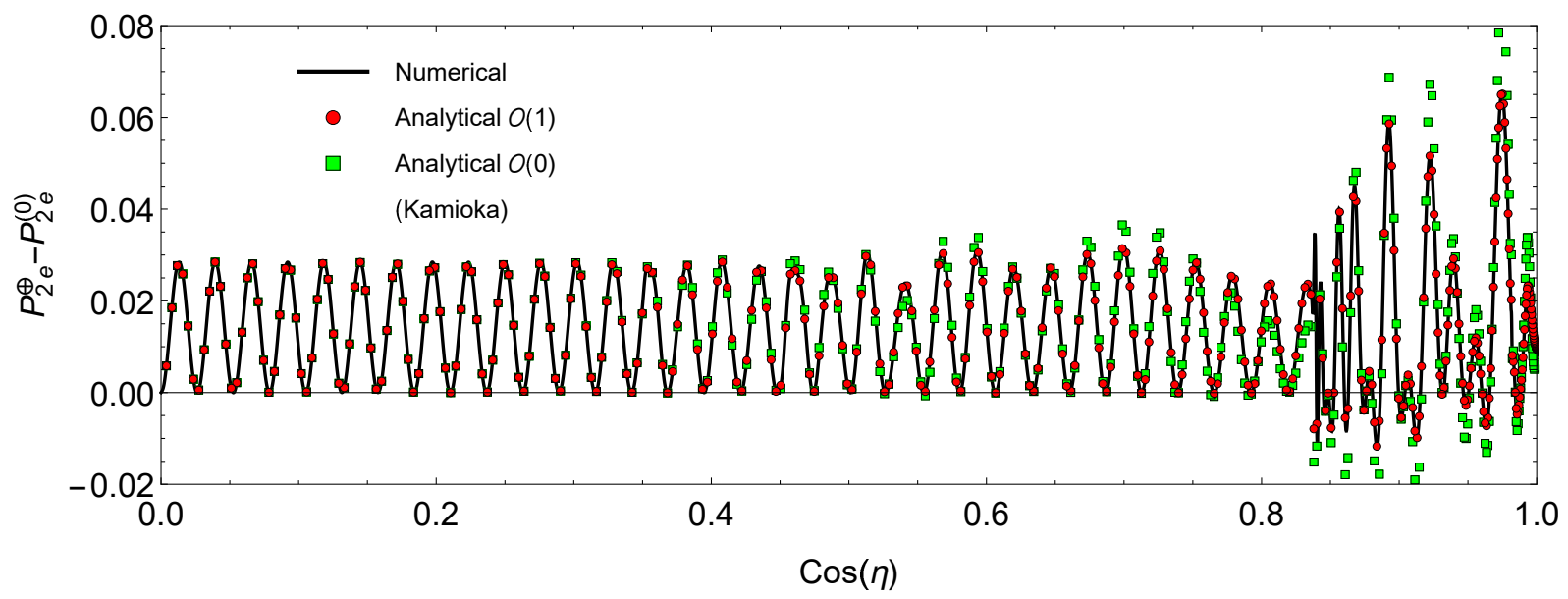

FIG. 2: Comparison of $P_{2 e}^{\oplus}-P_{2 e}^{(0)}$ versus $\cos \eta$ at $E=10 \mathrm{MeV}$. Solid curve represents the values obtained from numerical solution of evolution Eq. (13), whereas disk (red) and rectangular (green) marks represent the values obtained from our analytical expression with and without first order correction respectively. Oscillation parameters are taken of 3 neutrino LMA solution.

with first order correction nicely agree with numerical solution for all values of nadir angle. In Fig. 3, we plot nadir angle averaged value of $P_{2 e}^{\oplus}-P_{2 e}^{(0)}$ versus energy relevant for solar neutrinos. The detector location is assumed to be at Kamioka. The plot shows excellent agreement of analytical results (both with and without first order correction) with numerical solutions. This implies that treating the Earth density piecewise constant in five shells is a good approximation as far as calculations of averaged probability is concerned. The plot of Fig. 3 also shows that day-night asymmetry effect is very weak for solar neutrinos. The effect is not expected to be measured in SNO or BOREXINO experiments, as it is below their sensitivity [43].

In Fig. 4, we plot the conversion probability $P\left(v_{\mu} \rightarrow v_{e}\right)$ versus energy relevant for atmospheric and accelerator neutrinos. We produce three plots corresponding to three values of $\eta=0^{\circ}, 40^{\circ}$, and $80^{\circ}$. In each plot a comparison of analytical results (with and without first order correction) is made with the results obtained from numerical solutions. The plots show excellent agreement of first order corrected analytical results with the numerical solutions. A substantial increase in the probability observed about energy $E=5 \mathrm{GeV}$ is due to parametric enhancement well studied in Refs. [35 37]. As mentioned in Sec. II that the signs of electron density $N_{e}$ and $\mathrm{CP}$ phase $\delta_{C P}$ are inverted in the evolution equation 


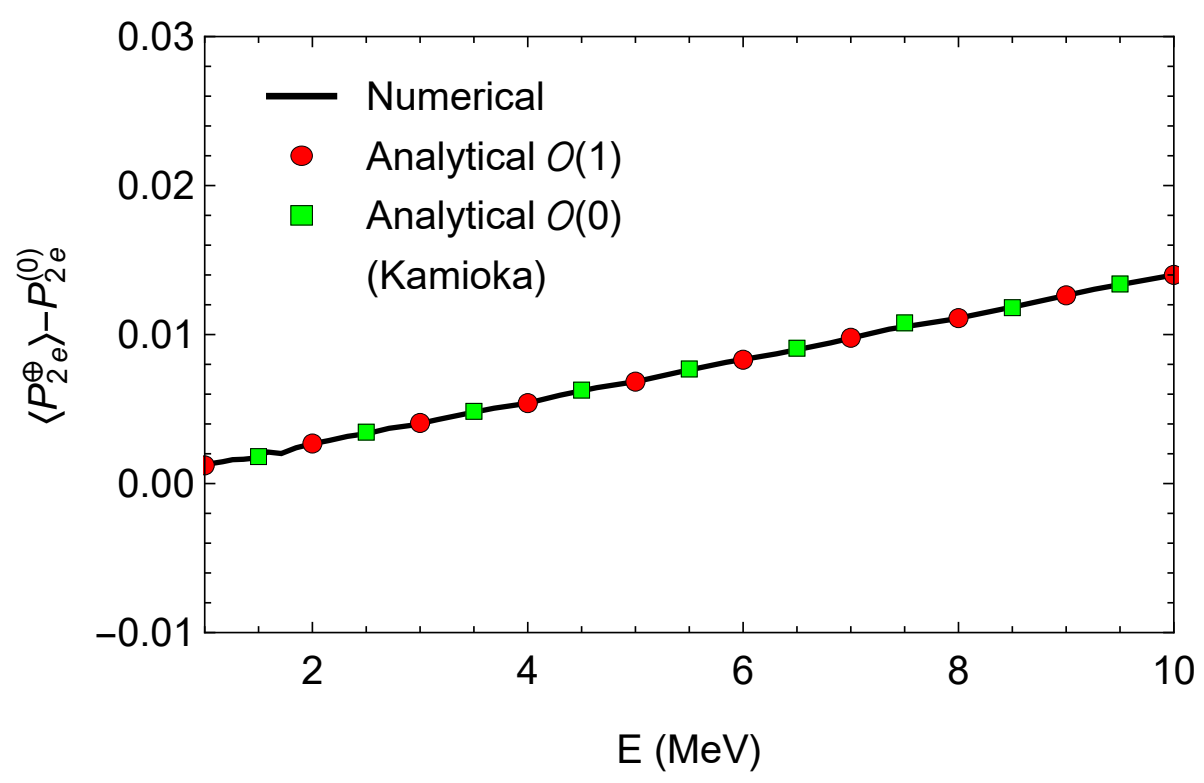

FIG. 3: Comparison of nadir angle averaged values of $P_{2 e}^{\oplus}-P_{2 e}^{(0)}$ versus energy relevant for solar neutrinos.

when applied on anti-neutrinos. As a result, the conversion probability $P\left(\bar{v}_{\mu} \rightarrow \bar{v}_{e}\right)$ becomes different from $P\left(v_{\mu} \rightarrow v_{e}\right)$. A part of this change is due to inverting the sign of density and a part due to inverting the sign of $\mathrm{CP}$ phase. In Fig. 5, we plot nadir angle averaged conversion probabilities $\left\langle P\left(v_{\mu} \rightarrow v_{e}\right)\right\rangle$ and $\left\langle P\left(\bar{v}_{\mu} \rightarrow \bar{v}_{e}\right)\right\rangle$ versus energy using our analytical expression. The plots clearly show a relative suppression in the probability $\left\langle P\left(\bar{v}_{\mu} \rightarrow \bar{v}_{e}\right)\right\rangle$. In the same figure we also plot the conversion probabilities assuming zero density. These plots (dashed line) show that a change of about 0.05 in the value of conversion probabilities of $v_{\mu} \rightarrow v_{e}$ and $\bar{v}_{\mu} \rightarrow \bar{v}_{e}$ is produced due to inverting the sign of CP phase in the energy range 0.5 to $10 \mathrm{GeV}$, if density is taken zero.

Recently T2K experiment [31] has measured CP phase through the study of difference between the conversion probabilities of $v_{\mu} \rightarrow v_{e}$ and $\bar{v}_{\mu} \rightarrow \bar{v}_{e}$ using accelerator neutrinos. In the base-line length of $295 \mathrm{~km}$ the expected change in the $P\left(v_{\mu} \rightarrow v_{e}\right)$ and $P\left(\bar{v}_{\mu} \rightarrow \bar{v}_{e}\right)$ probabilities is less than 0.025 for $E>0.4 \mathrm{GeV}$. More importantly, we find that the change is minimally sensitive to $\delta_{C P}$, which makes precise measurement of $\delta_{C P}$ difficult. It is noted that for base-line length $295 \mathrm{~km}$, the Earth matter effect is negligible. The sensitivity to CP phase is energy dependent and can also be affected by the Earth matter effect. This effect is highlighted in the Fig. 6, in which we plot the change in nadir angle averaged probabilities 
$\left\langle P\left(v_{\mu} \rightarrow v_{e}\right)\right\rangle$ and $\left\langle P\left(\bar{v}_{\mu} \rightarrow \bar{v}_{e}\right)\right\rangle$ versus energy for three different values of $\delta_{C P}$. The central curve correspond to $\delta_{C P}=1.45 \pi$; the best fitted value obtained by T2K experiment [31], whereas upper and lower curves correspond to the values $1.1 \pi$ and $1.8 \pi$, defined by measured error limits of $\delta_{C P}$ in T2K experiment. The plots clearly show that sensitivity to CP phase is indeed affected by Earth matter effect and in energy range 0.2 to $1 \mathrm{GeV}$, sensitivity to $\mathrm{CP}$ phase is maximum. In this energy range a variation of $\delta_{C P}$ in the range $1.1 \pi$ to $1.8 \pi$ can produce a variation of 0.1 in $\left\langle P\left(v_{\mu} \rightarrow v_{e}\right)\right\rangle-\left\langle P\left(\bar{v}_{\mu} \rightarrow \bar{v}_{e}\right)\right\rangle$. In order to study how the sensitivity to $\mathrm{CP}$ phase changes with nadir angle, we plot energy averaged probability difference $\left\langle P\left(v_{\mu} \rightarrow v_{e}\right)\right\rangle_{E}-\left\langle P\left(\bar{v}_{\mu} \rightarrow \bar{v}_{e}\right)\right\rangle_{E}$ versus nadir angle in Fig. 7. The energy averaged conversion probabilities $\left\langle P\left(v_{\mu} \rightarrow v_{e}\right)\right\rangle_{E}$ and $\left\langle P\left(\bar{v}_{\mu} \rightarrow \bar{v}_{e}\right)\right\rangle_{E}$ are calculated using Gaussian energy spectrum. The position of peak $E=0.6 \mathrm{GeV}$ and its width $\sigma \approx 0.3 \mathrm{GeV}$ are approximated from the energy spectrum of $v_{\mu}$ and $\bar{v}_{\mu}$ in T2K accelerator neutrino experiment [31]. In Fig. 7, we plot $\left\langle P\left(v_{\mu} \rightarrow v_{e}\right)\right\rangle_{E}-\left\langle P\left(\bar{v}_{\mu} \rightarrow \bar{v}_{e}\right)\right\rangle_{E}$ versus nadir angle corresponding to same three values of $\delta_{C P}$ used in Fig. 6. The plots show that sensitivity to $\delta_{C P}$ varies with nadir angle and it is maximum about $\cos \eta=0.3\left(\eta=72.5^{\circ}\right)$, which corresponds to base-line length $L=3827 \mathrm{~km}$. At the maxima, a variation of $\delta_{C P}$ in the range $1.1 \pi$ to $1.8 \pi$ can produce a variation of 0.07 in $\left\langle P\left(v_{\mu} \rightarrow v_{e}\right)\right\rangle_{E}-\left\langle P\left(\bar{v}_{\mu} \rightarrow \bar{v}_{e}\right)\right\rangle_{E}$. It is noted that this variation is merely 0.0022 at $\cos \eta=0.023\left(\eta=88.7^{\circ}\right)$; the nadir angle of T2K experiment. This suggests that an accelerator neutrino experiment constructed to work at base-line length $L \simeq 3872 \mathrm{~km}$ could be ideal for precise measurement of $\delta_{C P}$.

\section{Acknowledgments}

FA acknowledge the financial support of HEC through research grant 204500/NRPU/R\&D/HEC/14/727

[1] D. Davis, Jr., D.S. Harmer, and K.C. Hoffman, Phys. Rev. Lett. 20, 1205 (1968).

[2] B. Pontecorvo, Zh. Eksp. Teor. Fiz. 33, 549 (1957) and 34, 247 (1958); B. Pontecorvo, Zh. Eksp. Teor. Fiz. 53, 1717 (1967) [Sov. Phys. JETP 26, 984 (1968)].

[3] J.N. Abdurashitov et al., [SAGE Collab.], Phys. Rev. C80, 015807 (2009). 
(a)

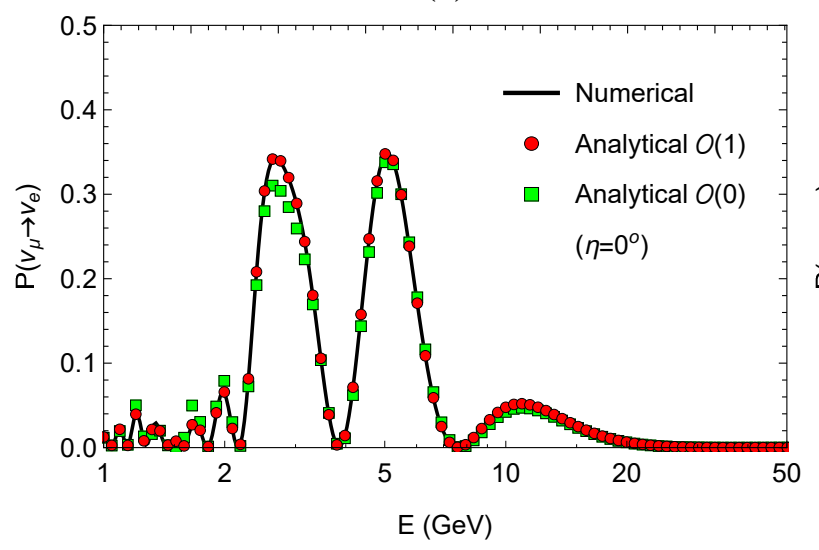

(b)

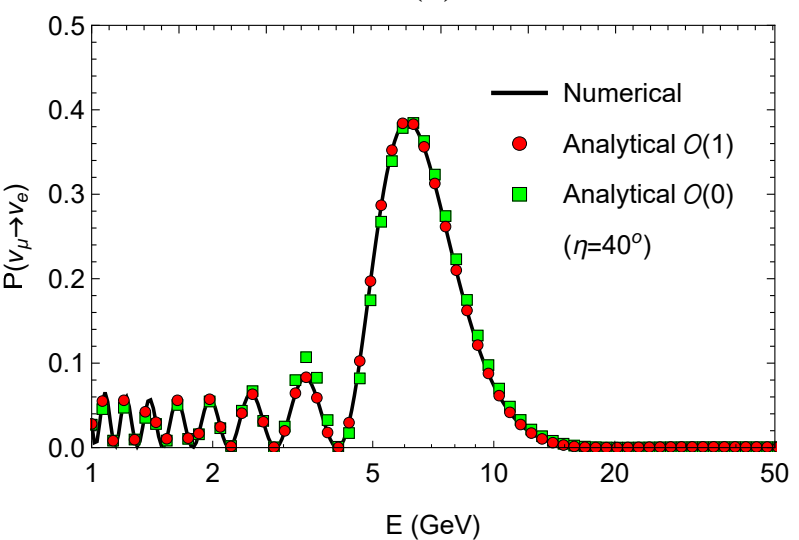

(c)

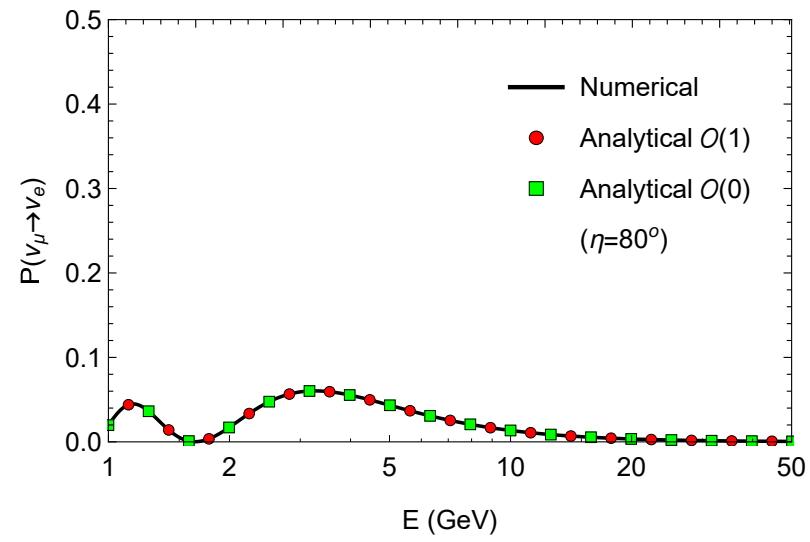

FIG. 4: Comparison of conversion probability $P\left(v_{\mu} \rightarrow v_{e}\right)$ versus energy relevant for atmospheric and accelerator neutrinos for three different values of nadir angle a) $\eta=0^{\circ}$, b) $\eta=40^{\circ}$, and c) $\eta=80^{\circ}$.

[4] P. Anselmann et al., [GALlEX Collab.], Phys. Lett. B285, 376 (1992); W. Hampel et al., [GALLEX Collab.], Phys. Lett. B447, 127 (1999).

[5] M. Altmann et al., [GNO Collab.], Phys. Lett. B616, 174 (2005).

[6] K.S. Hirata et al., [Kamiokande Collab.], Phys. Rev. Lett. 63, 16 (1989).

[7] Y. Fukuda et al., [Super-Kamiokande Collab.], Phys. Rev. Lett. 81, 1158 (1998);

[8] J. Hosaka et al., [Super-Kamiokande Collab.], Phys. Rev. D73, 112001 (2006).

[9] J.P. Cravens et al., [Super-Kamiokande Collab.], Phys. Rev. D78, 032002 (2008).

[10] K. Abe et al., [Super-Kamiokande Collab.], Phys. Rev. D83, 052010 (2011).

[11] K. Abe et al., [Super-Kamiokande Collab.], Phys. Rev. D94, 052010 (2016).

[12] B. Aharmim et al., [SNO Collab.], Phys. Rev. C72, 055502 (2005). 


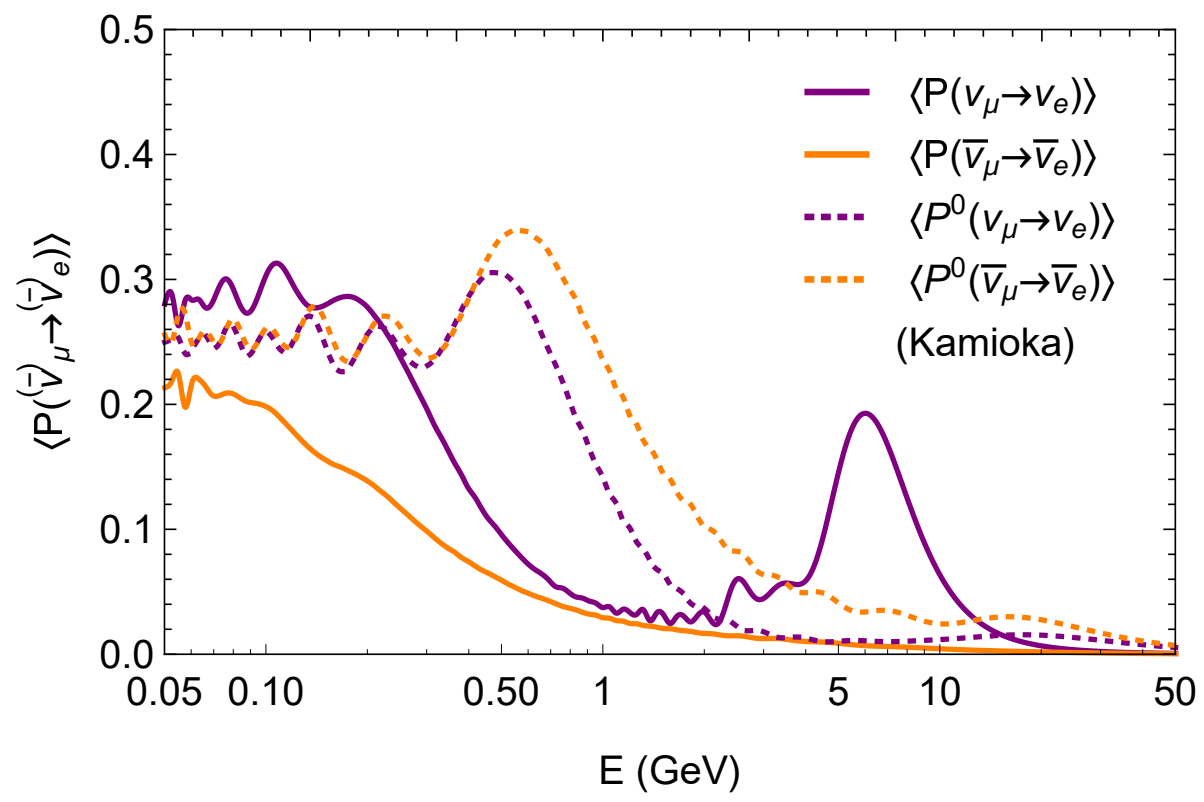

FIG. 5: Plots of nadir angle averaged survival probabilities $\left\langle P\left(v_{\mu} \rightarrow v_{e}\right)\right\rangle$ and $\left\langle P\left(\bar{v}_{\mu} \rightarrow \bar{v}_{e}\right)\right\rangle$ versus energy. Upper (purple) and lower (orange) solid curves represent probabilities $P\left(v_{\mu} \rightarrow v_{e}\right)$ and $P\left(\bar{v}_{\mu} \rightarrow \bar{v}_{e}\right)$ respectively. The dashed curves, produced for comparison, represent the same probabilities assuming zero density.

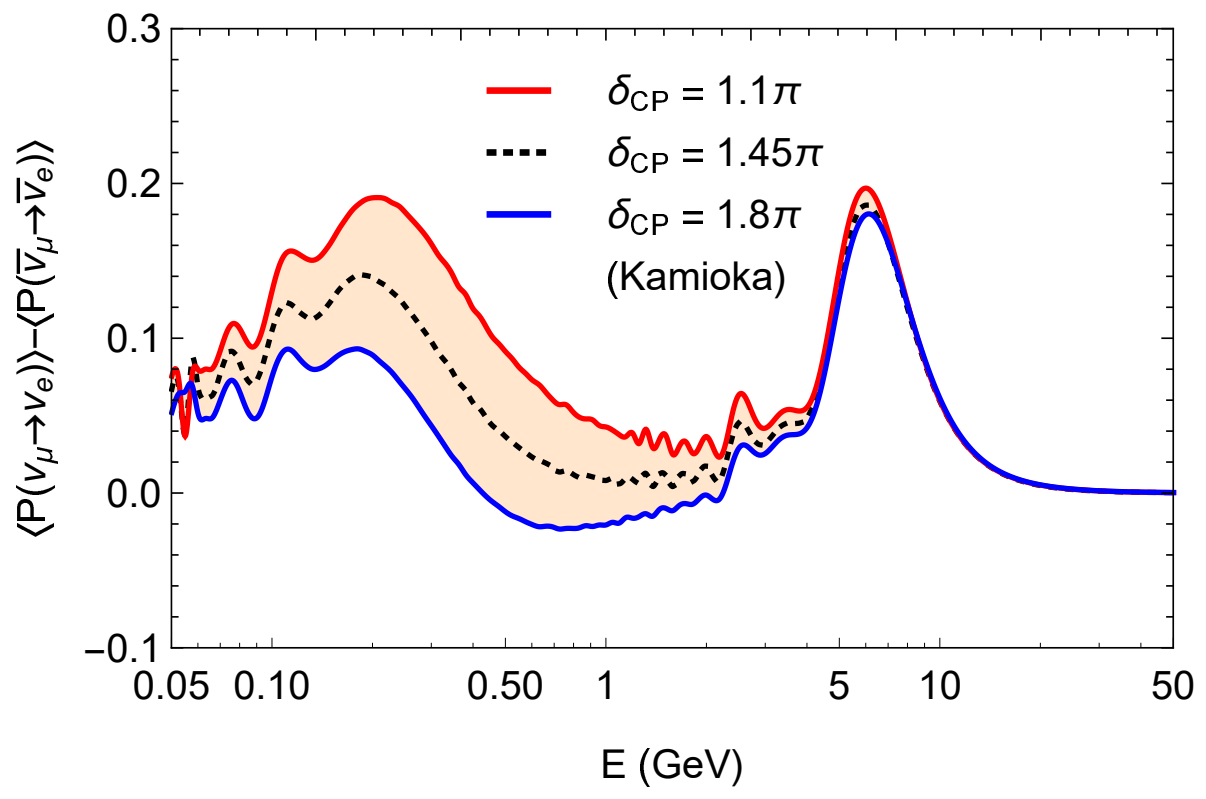

FIG. 6: Plots of nadir angle averaged probability difference $\left\langle P\left(v_{\mu} \rightarrow v_{e}\right)\right\rangle-\left\langle P\left(\bar{v}_{\mu} \rightarrow \bar{v}_{e}\right)\right\rangle$ versus energy for three different values of $\delta_{C P}$. For upper (red) and lower (blue) solid curves $\delta_{C P}$ is $1.1 \pi$ and $1.8 \pi$ respectively. 


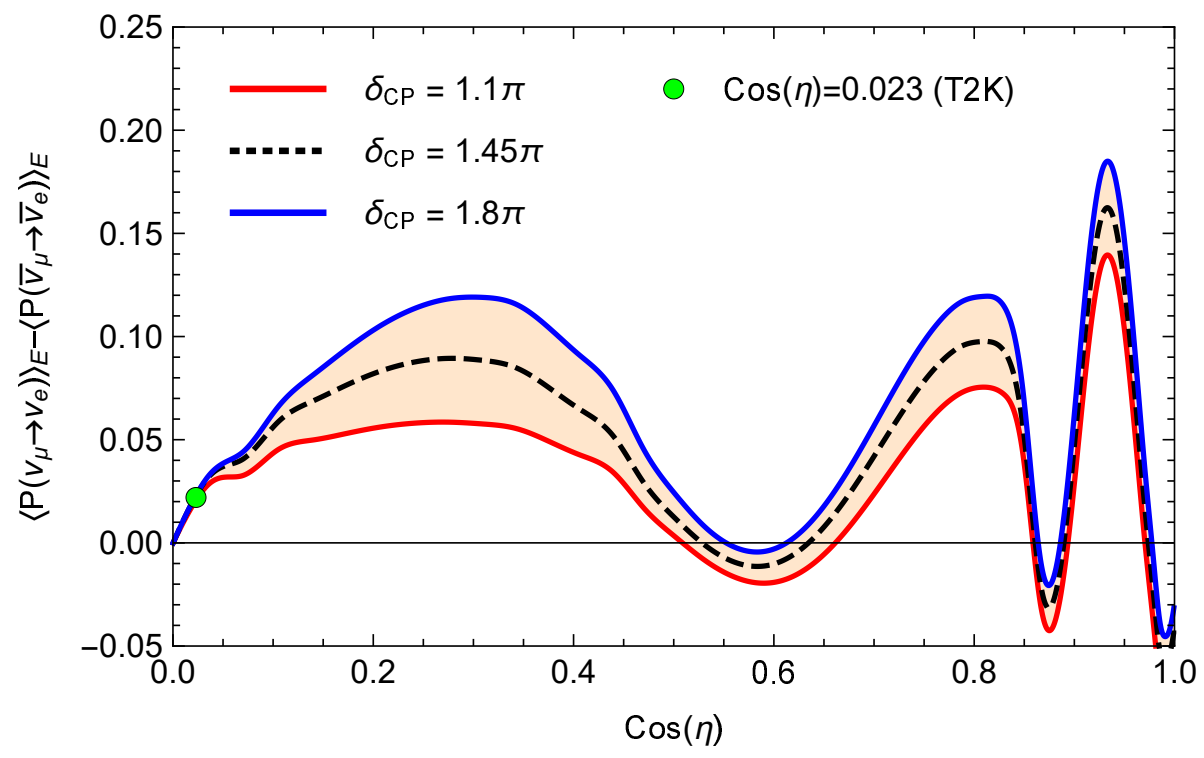

FIG. 7: Plots of energy averaged probability difference $\left\langle P\left(v_{\mu} \rightarrow v_{e}\right)\right\rangle_{E}-\left\langle P\left(\bar{v}_{\mu} \rightarrow \bar{v}_{e}\right)\right\rangle_{E}$ versus $\cos \eta$ for three different values of $\delta_{C P}$. The circular mark (green) is at $\cos \eta=0.023$, corresponding to base-line length $L=295 \mathrm{~km}$ of T2K experiment.

[13] B. Aharmim et al., [SNO Collab.], Phys. Rev. Lett. 101, 111301 (2008); Phys. Rev. C87, 015502 (2013).

[14] Z. Maki, M. Nakagawa, and S. Sakata, Prog. Theor. Phys. 28, 870 (1962).

[15] K. Abe et al. [Super-Kamiokande Collab.], Phys. Rev. D94 052010 (2016).

[16] S. Abe et al., [kamLAND Collab.], Phys. Rev. Lett. 100, 221803 (2008).

[17] Y. Fukuda et al., [Super-Kamiokande Collab.], Phys. Rev. Lett. 81, 1562 (1998).

[18] M. Ambrosio et al., [MACRO Collab.], Phys. Lett. B434, 451 (1998); Phys. Lett. B566, 35 (2003).

[19] M. Sanchez et al., [Soudan 2 Collab.], Phys. Rev. D68, 113004 (2003); W.W.M.

Allison et al., [Soudan 2 Collab.], Phys. Rev. D72, 052005 (2005).

[20] P. Adamson et al., [MINOS Collab.], Phys. Rev. D86, 052007 (2012).

[21] K. Abe et al., [T2K Collab.], Phys. Rev. D85, 031103 (2012); K. Abe et al., [T2K Collab.], Phys. Rev. Lett. 111, 211803 (2013).

[22] G. Tzanakos et al., [MINOS+ Collab.], FERMILAB-PROPOSAL-1016 (2011).

[23] K. Abe et al., [T2K Collab.], Phys. Rev. D85, 031103 (2012). K. Abe et al., [T2K Collab.], Phys. Rev. Lett. 111, 211803 (2013). 
[24] P. Adamson et al., [NOvA Collab.], Phys. Rev. D93, 051104 (2016).

[25] P. Adamson et al., [MINOS Collab.], Phys. Rev. Lett. 110, 171801 (2013).

[26] K. Abe et al., [T2K Collab.], Phys. Rev. Lett. 118, 151801 (2017).

[27] M. Tanabashi et al. (Particle Data Group), Phys. Rev. D 98, 030001 (2018).

[28] Y. Abe et al., [Double Chooz Collab.], Phys. Rev. Lett. 108, 131801 (2012).

[29] J.K. Ahn et al., [RENO Collab.], Phys. Rev. Lett. 108, 191802 (2012).

[30] F.P. An et al., [Daya Bay Collab.], Phys. Rev. Lett. 108, 171803 (2012).

[31] K. Abe et al. [T2K Collab.], Phys. Rev. D96, 092006 (2017).

[32] S.M. Bilenky, J. Hosek, and S.T. Petcov, Phys. Lett. B94, 495 (1980).

[33] L. Wolfenstein, Phys. Rev. D17, 2369 (1978).

[34] S.P. Mikheev and A.Y. Smirnov, Sov. J. Nucl. Phys. 42, 913 (1985); Nuovo Cimento 9C, 17 (1986).

[35] S.T. Petcov, Phys. Lett. B434, 321 (1998), (E) ibid. B444, 584 (1998).

[36] E.Kh. Akhmedov et al., Nucl. Phys. B542, 3 (1999).

[37] E.Kh. Akhmedov, Nucl. Phys. B538, 25 (1999).

[38] G. Bellini et al., [Borexino Collab.], Phys. Rev. Lett. 107, 141302 (2011).

[39] G. Bellini et al., [Borexino Collab.], Phys. Rev. Lett. 108, 051302 (2012).

[40] Eligio Lisi and Daniele Montanino, Phys. Rev. D56, 1792 (1997).

[41] Tommy Ohlsson and Hakan Snellman, J. Math. Phys. 41, 2768 (2000); Erratum: J. Math. Phys. 42, 2345 (2001).

[42] A. M. Dziewonski and D. L. Anderson, Phys. Earth Planet. Inter. 25, 297 (1981).

[43] E. K. Akhmedov, M. A. Tortola, J. W. F. Valle. JHEP 0405, 057 (2004). 